\title{
Clinical setting and extent of premortem evaluation do not predict autopsy discrepancy rates
}

\author{
Irini A Scordi-Bello ${ }^{1}$, Thomas H Kalb ${ }^{2}$ and Patrick A Lento ${ }^{1}$ \\ ${ }^{1}$ Department of Anatomic and Clinical Pathology, Mount Sinai School of Medicine, New York, NY, USA \\ and ${ }^{2}$ Division of Pulmonary, Department of Medicine, Critical Care and Sleep Medicine, Mount Sinai School \\ of Medicine, New York, NY, USA
}

\begin{abstract}
Autopsy rates have been affected by a number of factors, including technological advances and clinician beliefs of the diminished value of the autopsy. Such factors have resulted in a cultural shift in medicine away from the autopsy. Despite this shift, a number of studies have shown significant differences between antemortem clinical diagnoses and postmortem findings. Surveys of clinician beliefs about the autopsy have pointed toward antemortem diagnostic advancements as an important factor in declining rates. No study to date has addressed the hypothesis that such perceptions in diagnostic certainty have been matched by an actual decay in the yield of valuable or new information obtained by the autopsy. To address this hypothesis, we retrospectively compared the class I and class II discrepancies identified in $\mathbf{2 8 4}$ patients who died in three clinical settings with differing intensities of antemortem diagnostic workup. Despite a significantly different intensity of antemortem workup for patients in each clinical setting, including patients on a medical intensive care unit, patients on a surgical service and patients in an affiliated nursing home, discrepancy rates were found to be similar. Overall discrepancy rates for the medical intensive care unit, surgery service and nursing home patients were $27.8,32.7$ and $31.3 \%$, respectively $(P=0.84)$. In addition, we found no statistical difference in the complexity of workup in discrepant and nondiscrepant cases in each clinical setting. Our study data refute the hypothesis that the intensity of antemortem diagnostic evaluation correlates with an actual decrease in the rate of major diagnostic discrepancies identified at autopsy.

Modern Pathology (2010) 23, 1225-1230; doi:10.1038/modpathol.2010.107; published online 4 June 2010
\end{abstract}

Keywords: autopsy discrepancy; autopsy rates; class I and II; premortem evaluation

Hospital autopsy rates in the United States have declined from approximately $50 \%$ in the 1950 s to $5-10 \%$ in the 1990 s. $^{1}$ Multiple factors, including the elimination of the hospital autopsy rate requirement by the Joint Commission for the Accreditation of Hospitals in 1972, have contributed to this dramatic decline. Other frequently cited factors include the lack of reimbursement for the autopsy, clinician concerns about litigation, cultural and religious objections by families, and increasing workload burden for house officers.

An additional factor was highlighted by a recent survey of medical students, physician trainees and practicing physicians, pointing to a decay in the

Correspondence: Dr IA Scordi-Bello, MD, PhD, Department of Anatomic and Clinical Pathology, Mount Sinai School Of Medicine, One Gustave Levy Place, New York, NY 10029, USA. E-mail: irini.scordibello@mssm.edu

Received 4 January 2010; revised 8 March 2010; accepted 12 March 2010; published online 4 June 2010 priority assigned to the autopsy as a clinical teaching tool. ${ }^{2}$ For the busy house officer who is typically relegated to the task of obtaining consent, the time and effort dedicated to this burdensome task may be affected if they perceive that no additional knowledge or little clinically relevant information will be gained from the autopsy. As a consequence, the widely held belief by those physicians surveyed, that antemortem diagnosis is rarely altered by postmortem examination, may be a significant contributor to the continued decline of autopsy rates. This has lead to a cultural shift in Medicine that devalues the task of obtaining autopsy consent and the value assigned to the autopsy in general.

A reasonable hypothesis is that this widely held perception is based on real advances in antemortem diagnostic certainty, given the explosive growth of medical technology and information. Indeed, advances in imaging, clinical laboratory studies, and surgical and nonsurgical tissue sampling techniques 
over the past several decades have dramatically influenced the quality of antemortem evaluation, helping to define the location and extent of disease and yielding a greater number of antemortem diagnoses. Such advances may have undoubtedly contributed to shifting physician attitudes toward the autopsy, though it is not clear whether this attitude is matched by actual observed decay in the yield of valuable or new information obtained by postmortem examination. ${ }^{3}$

The landmark study by Goldman et $a l^{4}$ in 1983 showed, however, that despite the advances in medicine discrepancy rates between clinical diagnoses and autopsy findings were similar over a period of three decades (1960-1980). In the autopsy cases assessed in this study, about $10 \%$ of autopsies revealed a major diagnosis that might have led to a change in therapy and/or prolonged survival had the diagnosis been known before death. ${ }^{5}$ Other studies since then have shown similar results with discrepancy rates ranging from 8 to more than $30 \%$, depending on the clinical setting. ${ }^{5-11}$

No study to date has adequately addressed the hypothesis that the intensity and the extent of antemortem diagnostic evaluation correlate with an actual decrease in the number of unexpected discrepancies at autopsy. We retrospectively reviewed all adult autopsies performed at a tertiary care center over a 4-year period to assess whether the discrepancy rate correlated with an index of diagnostic intensity. We also assessed whether patients in clinical settings with a higher intensity of antemortem evaluation, such as the intensive care unit or surgical service, had lower discrepancy rates than nursing home patients, who died without antemortem hospitalization and with minimal antemortem evaluation.

\section{Materials and methods}

We retrospectively reviewed all adult autopsy cases $(n=562)$ performed at our institution over a 4-year period (1 January 2003 to 31 December 2006) to determine the discrepancy rate between clinical diagnoses and autopsy diagnoses. All autopsies were performed by pathology residents at our program under the direct supervision of attending pathologists. Cases were divided into three clinical groups: (1) patients on the surgical service that had recently undergone surgery or had been readmitted after surgery and were under the care of a surgical team at the time of death, including patients in the surgical intensive care unit; (2) patients from the medical intensive care unit and (3) patients who died at two affiliated local nursing homes. Only cases that fell into one of the three clinical patient groups noted were included in our study.

Clinical diagnoses were extracted from reviews of patient medical records performed at the time of death, accompanying death certificates filled out by
Table 1 Antemortem workup scoring system

\begin{tabular}{ll}
\hline Points & Representative tests \\
\hline 1 & Routine blood work: urinalysis, blood/urine cultures \\
2 & $\begin{array}{l}\text { Simple radiology: CXR, AXR, KUB, obstructive } \\
\text { series, ultrasound }\end{array}$ \\
3 & Advanced radiology: CT scan, CT angiogram, \\
& MRI/MRA, V/Q scan \\
& Invasive procedures: surgery, fine needle aspiration, \\
& bronchoalveolar lavage, bone marrow biopsy, liver \\
& biopsy \\
& 0-3 points = low complexity \\
& 4-6 points = intermediate complexity \\
& 7-10 points = high complexity
\end{tabular}

the clinical staff and, when possible, discussions with the clinical staff. These diagnoses were compared with autopsy findings in respective cases, which were obtained from finalized autopsy reports maintained in the Department of Pathology. Assessment of the extent of antemortem diagnostic evaluation for each patient was compiled from chart reviews and electronic hospital medical records. A patient's antemortem workup was scored using a system we devised (see Table 1). Higher scores were given to more complex and/or invasive tests, including surgical and biopsy procedures that yielded tissue or other material for pathologic diagnosis, and lower scores were given to routine, noninvasive procedures, such as blood work and chest X-rays. Tests were only counted once toward the total score (ie if a patient had a chest X-ray performed every day of a 20-day hospital stay, only 2 points for the test were assigned to the total score). Patients were then categorized into three general groups according to the total score of all procedures (low, intermediate and high complexity workup).

Autopsy discrepancies were classified into two broad categories, according to the Goldman criteria. Only class I and class II discrepancies were included our study. Class I discrepancies included major diagnoses that, if detected before death, could have influenced management with potential prolonged survival. Class II discrepancies included major diagnoses that would not have altered management.

Statistical analysis was performed using the SPSS software version 15 (SPSS Inc., Chicago, IL, USA). Categorical variables were compared using the Pearson's $\chi^{2}$-test and $P$-values $<0.05$ were considered significant.

\section{Results}

Over a 4-year period, 562 adult autopsies were performed at our institution. Of them, 284 cases fell into one of the three clinical groups being assessed and were included in our study (97 surgery, 107 medical intensive care and 80 nursing home cases). The hospital autopsy rate ranged from 10 to $12 \%$ 
during the 4-year period being studied. The nursing home autopsy rate was not available.

The level of diagnostic workup was significantly different in each of the three clinical settings studied (Figure 1). The majority of surgical patients $(77 \%)$ received complex diagnostic tests, whereas 38\% of medical intensive care unit patients and only $3 \%$ of nursing home patients scored in this 'high-complexity' category $(P<0.0005)$. Medical nursing home patients $(91 \%)$ received only basic, routine tests and scored in the 'low-complexity' category.
The discrepancy rates between clinical and postmortem diagnoses in these three settings were very similar. The overall discrepancy rates for the surgery, medical intensive care and nursing home settings were 28, 32 and $31 \%$, respectively $(P=0.84)$. Class I discrepancy rates for the surgery, medical intensive care and nursing home groups were 19,19 and $21 \%$, respectively $(P=0.91)$. Similarly, class II discrepancy rates were 9, 13 and $10 \%$, respectively, with no statistically significant differences $(P=0.7)$ (Figure 2). Diagnostic

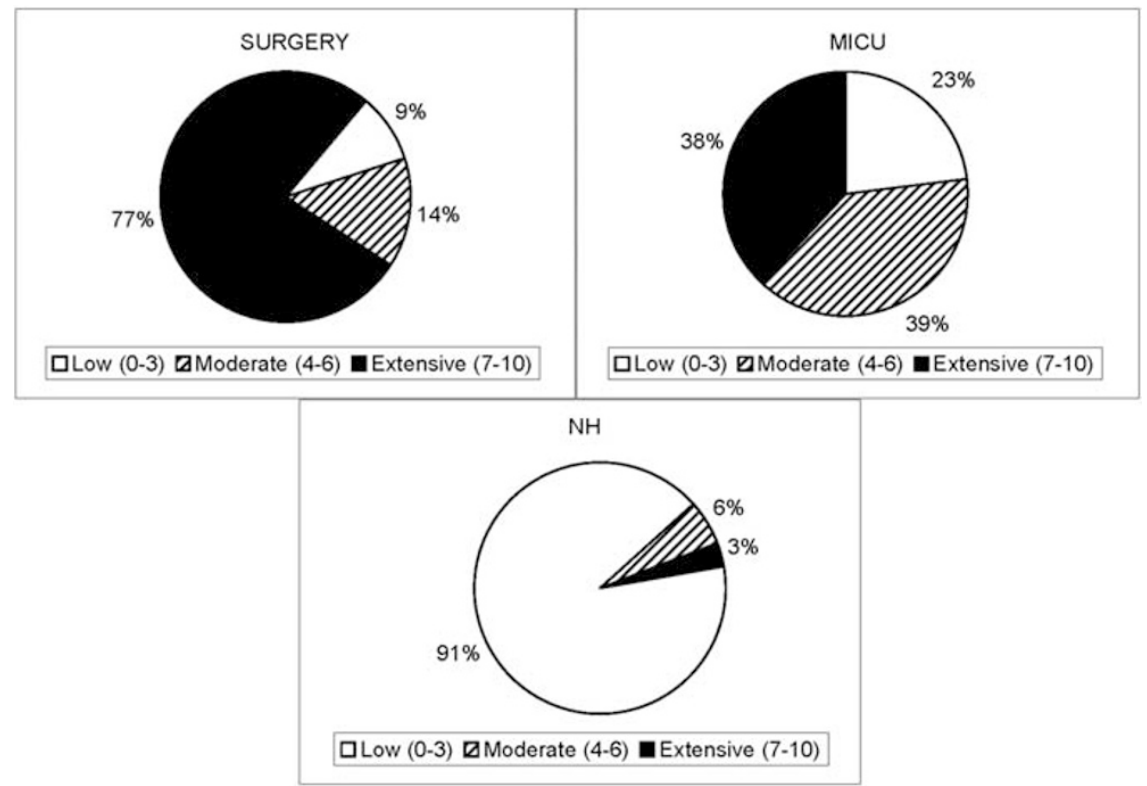

Figure 1 The level of diagnostic workup differs depending on the clinical setting.

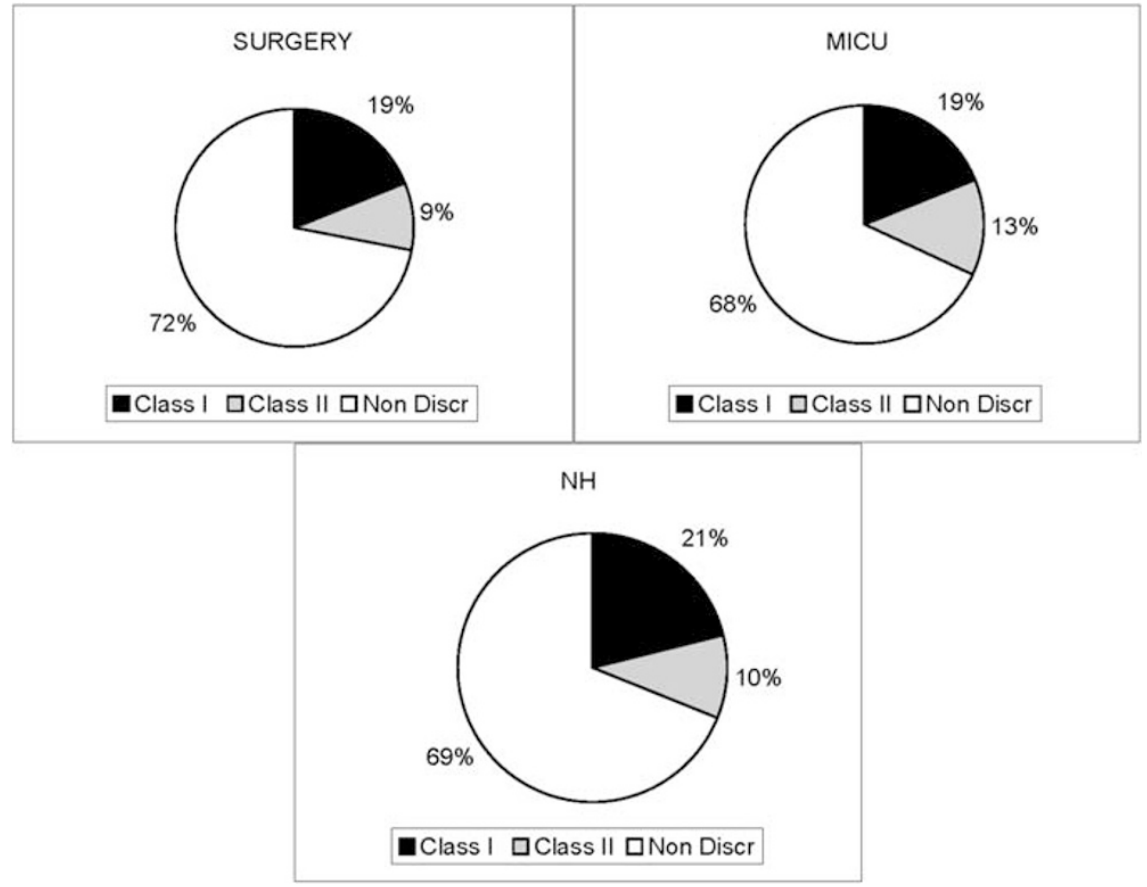

Figure 2 Discrepancy rates are similar independent of clinical setting. 


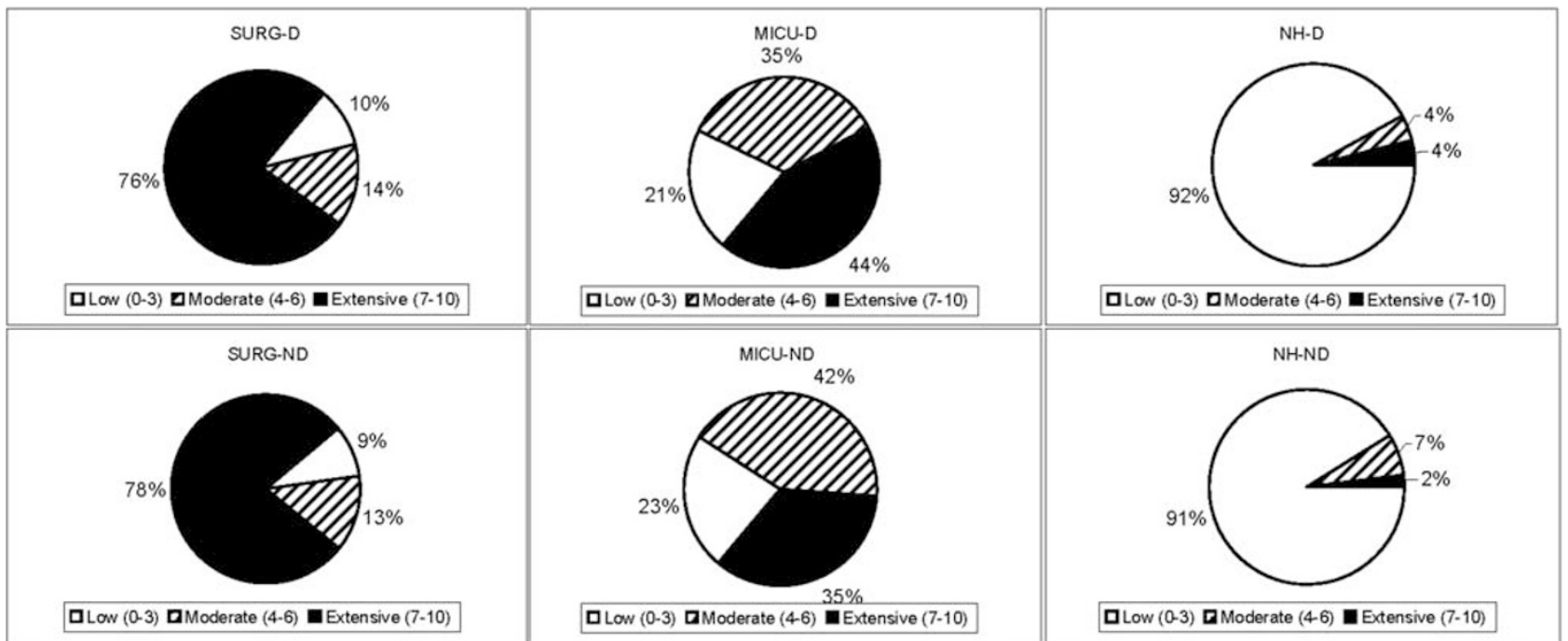

Figure 3 Discrepant and nondiscrepant cases in each clinical setting do not differ in the level of antemortem evaluation.

discrepancy did not correlate with age $(P=0.265)$, sex $(P=0.425)$ or year of death $(P=0.937)$.

Within each clinical setting, cases with discrepancies were compared with those without discrepancies about the extent of antemortem testing to see whether the extent of testing was significantly different between these two groups. We determined that discrepant and nondiscrepant cases had similar profiles regarding complexity of antemortem workup (Figure 3 ). In the surgery group, $76 \%$ of cases in the discrepant group had undergone extensive testing, compared with $78 \%$ of cases in the nondiscrepant group $(P=0.927)$. Similarly in the medical intensive care group, $44 \%$ of discrepant cases had undergone extensive testing, which was not significantly different from the $35 \%$ of nondiscrepant cases $(P=0.721)$. In the nursing home group, $4 \%$ of discrepant cases had extensive workup compared with $2 \%$ in the nondiscrepant cases $(P=0.859)$.

The types of discrepancies in each setting were classified in six broad categories and are shown in Table 2. The surgical group had the most diverse list of diagnoses that were newly detected at autopsy. Infections, gastrointestinal, and hepatobiliary and pulmonary complications each comprised $18 \%$. Cardiovascular complications, such as myocardial infarcts, aneurysms, dissections and cholesterol embolization, comprised $14 \%$, whereas missed malignancies comprised only $7 \%$. Twenty-five percent of missed diagnoses were perioperative and postoperative complications, technical failures and therapeutic complications not known before death.

Most diagnoses undetected before autopsy in the medical intensive care setting were infectious in nature $(42 \%)$, most notably fungal infections, followed by various gastrointestinal and hepatobiliary complications (32\%).

The most common undetected diagnosis in the nursing home setting was pulmonary embolism
(48\%), whereas gastrointestinal and hepatobiliary complications and undiagnosed malignancies each represented $16 \%$ of discrepancies. Of note, myocardial infarcts comprised only $8 \%$ and infections $12 \%$ of the total missed diagnoses.

\section{Discussion}

This study has confirmed that a substantial number of important diagnoses not detected before death are revealed by autopsy and, most notably, that the rate of such undetected diagnoses was unaltered by the intensity of antemortem evaluation. In fact, no difference was seen in the rate of important diagnostic discrepancies detected between patients who died in a nursing home and those who underwent more extensive evaluation in a hospital including, at times, surgery and/or biopsy in a surgical or intensive care unit setting. This study provides new information that belies the notion that advances in technology, invasive testing and diagnostic armamentarium have led to a decay in the information gained at autopsy.

Unfortunately, recent elimination of an autopsy requirement from the Accreditation Council for Medical Education (ACGME) program requirements for Residency Education in Internal Medicine highlights the growing shift away from the autopsy. The continued requirement of the performance of autopsies for residency training in Pathology may expose misaligned agendas of ACGME components, in part fueled by a misperception that has reached the level of pedagogic authority and policy statements, though one that is not supported by these findings.

The landmark autopsy study by Goldman et $a l^{4}$ (1983) highlighted the historically recognized importance of the autopsy over a three decade period in identifying discrepancies between clinical (ie 
Table 2 Summary of diagnostic discrepancies

\begin{tabular}{|c|c|c|c|}
\hline & Surgery & $M I C U$ & Nursing home \\
\hline Infections & $\begin{array}{l}\text { Infectious Endocarditis } \times 2 \\
\text { Infected SVC/RA thrombus } \\
\text { Chronic endocarditis } \\
\text { Fungal pericarditis }\end{array}$ & $\begin{array}{l}\text { Invasive pulmonary aspergillus } \times 5 \\
\text { Systemic histoplasma } \\
\text { Disseminated P. multocida } \\
\text { Disseminated Candida } \\
\text { Adenovirus pneumonia } \\
\text { Bacterial endocarditis } \\
\text { Disseminated mucor } \\
\text { Group A } \beta \text {-hemolytic Strep } \\
\text { Stenotrophomonas maltophilia }\end{array}$ & $\begin{array}{l}\text { Subacute bacterial } \\
\text { endocarditis } \\
\text { Bronchopneumonia }\end{array}$ \\
\hline Pulmonary & Pulmonary emboli $\times 5$ & $\begin{array}{l}\text { Pulmonary fibrosis c/w UIP } \\
\text { UIP exacerbation } \\
\text { Pulmonary embolus }\end{array}$ & $\begin{array}{l}\text { Pulmonary emboli } \times 10 \\
\text { DVT subclavian/axillary } \\
\text { Recurrent aspirations/fibrosis } \\
\text { Aspiration pneumonia }\end{array}$ \\
\hline Cardiovascular & $\begin{array}{l}\text { Acute and chronic myocardial } \\
\text { infarcts } \\
\text { Cholesterol embolization } \\
\text { Ruptured AAA } \\
\text { Acute aortic dissection }\end{array}$ & & $\begin{array}{l}\text { Acute myocardial infarct } \\
\text { Acute myocardial infarct } \\
\text { with rupture }\end{array}$ \\
\hline Hepatobiliary/GI & $\begin{array}{l}\text { Obstructive choledocholithiasis } \\
\text { Ileosigmoid knotting } \\
\text { Xanthogranulomatous cholecystitis } \\
\text { Acalculous cholecystitis } \\
\text { Splenic rupture }\end{array}$ & $\begin{array}{l}\alpha-1 \text { antitrypsin cirrhosis } \\
\text { Ischemic bowel with perforation } \\
\text { Kayexelate necrosis-bowel perforation } \\
\text { Ruptured diaphragmatic varices } \\
\text { Renal vein/IVC thrombosis } \\
\text { Acute peritonitis-gastric leak } \\
\text { Small bowel strangulation } \\
\text { Hepatic veno-occlusive disease } \\
\text { Hepatitis C cirrhosis and HCC UGIB } \\
\text { Nodular regenerative hyperplasia } \\
\text { (NRH) } \\
\text { Hepatic venous outflow obstruction }\end{array}$ & $\begin{array}{l}\text { Perforated duodenal ulcer } \\
\text { Dehiscence of anastomosis } \\
\text { Duodenal ulcer with UGIB } \\
\text { Portal vein thrombosis and } \\
\text { granulomatous cirrhosis }\end{array}$ \\
\hline Malignancy & $\begin{array}{l}\text { Metastatic adenoCa, unknown } \\
\text { primary } \\
\text { Metastatic gastric adenoCa }\end{array}$ & $\begin{array}{l}\text { Ovarian carcinoma } \\
\text { Peripancreatic gastrinoma (ZE) } \\
\text { Metastatic pancreatic adenoCa } \\
\text { Metastatic carcinoma of UO } \\
\text { Hep C with cirrhosis and HCC }\end{array}$ & $\begin{array}{l}\text { Mucinous adenocarcinoma } \\
\text { Cervical adenosquamous Ca } \\
\text { Gastric GIST } \\
\text { Extensive breast cancer } \\
\text { metastasis }\end{array}$ \\
\hline $\begin{array}{l}\text { Therapeutic } \\
\text { complications }\end{array}$ & $\begin{array}{l}\text { Aortic root dissection } \\
\text { Periaortic hematoma, prox arch } \\
\text { Transmural aortic ulcer } \\
\text { Right hemothorax/IC artery } \\
\text { Transmural LA tear } \\
\text { Esophageal tear/RESCA } \\
\text { Hepatic artery necrosis } \\
\text { hemoperitoneum }\end{array}$ & Neurodegenerative disease & \\
\hline
\end{tabular}

antemortem) diagnoses and autopsy findings. More recent studies since then have cited a range of discrepancies in varied clinical settings. Combes et $a 1^{11}$ reported a $31.7 \%$ discrepancy rate (class I and class II) in an intensive care population whereas Pastores et $a l^{6}$ reported a $26 \%$ discrepancy rate in a group of critically ill patients in an oncology intensive care unit. Mort and Yeston ${ }^{10}$ described a major discrepancy rate of $23 \%$ in a surgical intensive care unit. Recently, Tavora et al reported an overall discrepancy rate of $17.2 \%$ in three institutional settings, which included a university hospital, a community hospital and a private autopsy service. In a systematic review of the literature by Shojania et $a l,{ }^{12}$ the authors reported a relative decrease in important unsuspected diagnoses over a 40-year period; however, the possibility that an autopsy would reveal a missed diagnosis still remained sufficiently high to warrant the ongoing use of the autopsy.

Despite these and other data, autopsy rates have continued to decline and the cultural shift in medicine away from the autopsy has grown. This shift away from the autopsy has been fueled, in part, by a widely held belief of surveyed clinicians that current advances in medicine have rendered the autopsy obsolete or of reduced value. Our study has addressed one potential component of this perception by testing the hypothesis that the intensity/ complexity of antemortem diagnostic evaluation correlates with an actual decrease in the number of discrepancies at autopsy. We retrospectively compared major (class I and class II) discrepancy rates in three separate clinical settings over a 4-year period. Using a scoring system we devised, we then compared the complexity/intensity of antemortem 
diagnostic evaluation among the three clinical settings to see if higher intensity diagnostic evaluation had an impact on reducing autopsy discrepancies.

As expected, the complexity score for antemortem testing was highest for patients on the surgical service, intermediate for those in the medical intensive care unit and lowest for nursing home patients. Despite differences in the intensity of antemortem workup, discrepancy rates were not significantly different in the three settings. In addition, we found that the level of antemortem diagnostic evaluation did not differ between discrepant and nondiscrepant cases within each clinical setting. Cases with extensive antemortem testing were just as likely to have a missed diagnosis identified at autopsy as those without such a workup.

Our study is limited by its nonrandomized retrospective design. As a result, it is unclear whether the subset of patients who underwent autopsy was different than others who died in these different practice settings who were not autopsied. One can also not ignore the possibility that some level of diagnostic uncertainty or suspicion of missed diagnoses led to a greater effort on the part of those obtaining successful autopsy consent. As such, caution must be applied to extrapolating the rate of discrepancies to the overall medical population. Also, our scale of intensity of antemortem evaluation does not account for physician suspicions, nor for diagnostic studies that were considered or warranted but not performed because of technical, physical or consent limitations.

Nonetheless, these data refute the hypothesis that the intensity and/or complexity of antemortem diagnostic evaluation correlates with an actual decrease in the number of discrepancies at autopsy and support the continued value of the autopsy in spite of medical diagnostic advancements. Because the autopsy also provides for quality assurance of care rendered, enhances the accuracy of death certification, aids in the identification of emerging or reemerging pathogens/diseases and serves as an educational format for clinicians, pathologists and students alike in the understanding of disease, one could argue, in fact, that more autopsies are warranted in the face of newer medical techniques.

\section{Disclosure/conflict of interest}

The authors declare no conflict of interest.

\section{References}

1 Hoyert DL, Kung HC, Xu J. Autopsy patterns in 2003. National Center for Health Statistics. Vital Health Stat 2007;20:1-27.

2 Hooper JE, Geller SA. Relevance of the autopsy as a medical tool: a large database of physician attitudes. Arch Pathol Lab Med 2007;131:268-274.

3 Horowitz RE, Naritoku WY. The autopsy as a performance measure and teaching tool. Hum Pathol 2007;38:688-695.

4 Goldman L, Sayson R, Robbins S, et al. The value of the autopsy in three medical eras. $N$ Engl J Med 1983;308:1000-1005.

5 Tavora F, Crowder CD, Sun CC, et al. Discrepancies between clinical and autopsy diagnoses: a comparison of university, community, and private autopsy practices. Am J Clin Pathol 2008;129:102-109.

6 Pastores SM, Dulu A, Voigt L, et al. Premortem clinical diagnoses and postmortem autopsy findings: discrepancies in critically ill cancer patients. Crit Care 2007;11:R48.

7 Burton EC, Troxclair DA, Newman III WP. Autopsy diagnoses of malignant neoplasms: how often are clinical diagnoses incorrect? JAMA 1998;280: 1245-1248.

8 Perkins GD, McAuley DF, Davies S, et al. Discrepancies between clinical and postmortem diagnoses in critically ill patients: an observational study. Crit Care 2003;7:R129-R132.

9 Saad R, Yamada AT, Pereira da Rosa FH, et al. Comparison between clinical and autopsy diagnoses in a cardiology hospital. Heart 2007;93:1414-1419.

10 Mort TC, Yeston NS. The relationship of pre mortem diagnoses and post mortem findings in a surgical intensive care unit. Crit Care Med 1999;27:299-303.

11 Combes A, Mokhtari M, Couvelard A, et al. Clinical and autopsy diagnoses in the intensive care unit: a prospective study. Arch Intern Med 2004;164: 389-392.

12 Shojania KG, Burton EC, McDonald KM, et al. Changes in rates of autopsy-detected diagnostic errors over time: a systematic review. JAMA 2003;289: 2849-2856. 